\title{
A73 MIR-155 AND MIR-34A REGULATE PROINFLAMMATORY CYTOKINE PRODUCTION BY HUMAN MONOCYTES
}

M Kurowska-Stolarska, L Ballantine, B Stolarski, J Hunter, A Hueber, J A Gracie, FY Liew, I B Mclnnes Glasgow Biomedical Research Centre, University of Glasgow, UK

10.1136/ard.2010.129619g

Purpose Dysregulated proinflammatory activation of synovial monocytes and macrophages is of fundamental importance in the pathogenesis of rheumatoid arthritis (RA). Recently, microRNAs (miRNA) have been identified as critical posttranscriptional regulators of many components of immune responses. The aim of our study was to investigate the role of candidate miRNAs miR-155 and miR-34a in the activation of RA synovial fluid (SF) and blood monocyte/macrophages.

Methods CD14 cells from SF or blood of RA patients and CD14 cells from blood of healthy controls were isolated using CD14 MACS MicroBeads. Peripheral blood (PB) CD14 cells were stimulated with lipopolysaccharide $(100 \mathrm{ng} / \mathrm{ml})$, Pam3CSK4 (300 ng/ml), Polyl:C (50 $\mathrm{g} / \mathrm{ml})$, CPG $(3 \mu \mathrm{g} / \mathrm{ml})$ or control CPG $(3 \mu \mathrm{g} / \mathrm{ml})$. PB CD14 cells were transfected with miR-155, miR-34a and scramble mimics (all $3.5 \mu \mathrm{g} / 3 \times 10^{6}$ cells) by electroporation. Total RNA was isolated by miRNeasy kit. TaqMan miRNA and mRNA assays were used for semi-quantitative determination of the expression of miR-155, miR-34a and AXL, respectively. The expressions of U6B small nuclear RNA and ribosomal $18 \mathrm{~S}$ or $\beta$-actin were used as endogenous controls. To identify the cellular targets of candidate miRs, multiple target prediction programmes and mRNA transcriptomic signatures of SF CD14 cells were employed.

Results SF CD14 cells expressed higher levels of miR-155 (64-fold, $\mathrm{p}<0.05$ ) and miR-34a (4.5-fold, $\mathrm{p}<0.05)$ than blood matched CD14 cells. Expression of miR-155 was upregulated by TLR2, TLR3, TLR4 and TLR9 ligands $(\mathrm{p}<0.05)$ in PB CD14 cells. In contrast, miR-34a expression was either not affected (Pam3CSK4) or downregulated (LPS, PolyI:C, CPG; $\mathrm{p}<0.05$ ) under the same conditions. Overexpression of miR-155 and miR-34a mimics in blood monocytes induced tumour necrosis factor $\alpha($ TNF $\alpha)$ production $(10170 \pm 385 \mathrm{pg} / \mathrm{ml})$ and $(991 \pm 33 \mathrm{pg} /$ $\mathrm{ml})$, respectively. In addition, overexpression of miR-155 but not miR-34a in human PB monocytes increased interleukin 6 (IL-6) production. Using computational target ranking system combined with mRNA transcriptomic data, the authors identified a negative regulator of TLR/IL-1R signalling, AXL (tyrosine kinase receptor) to be targeted by both miRs. Overexpression of miR-34a and miR-155 mimics decreased the expression of AXL in blood monocytes by $48 \pm 3 \%$ and $38 \pm 16 \%$, respectively.

Conclusion These data suggest that overexpression of miR155 and miR-34a in CD14 cells can result in downregulation of AXL expression which in turn can lead to the overproduction of TNF $\alpha$. The authors provide a novel mechanism for deregulated TNF production in RA synovial macrophages amenable to therapeutic intervention in due course. 